\title{
Extended Emission-Line Regions Around AGN
}

\author{
A. Robinson \\ Department of Physical Sciences, University of Hertfordshire, Hatfield, \\ Hertfordshire AL10 $9 A B, U K$
}

\begin{abstract}
High-excitation extended emission-line nebulae around active galactic nuclei probably result either from photoionization of the interstellar medium by beamed continuum radiation, or dynamical interactions between radio sources and the ambient gas, or both. Here I discuss the models based on these mechanisms, and their relative importance in radio-quiet and radio-loud active galaxies.
\end{abstract}

\section{Introduction}

Extended emission-line regions (EELR) are found around many active galactic nuclei, covering a wide range of spatial scales and typically exhibiting a highexcitation spectrum. If we interpret 'extended' as 'spatially resolved', these nebulae range in size from the narrow-line region in nearby Seyfert nuclei, where $H S T$ images reveal structure on scales of $10-100 \mathrm{pc}$, to vast line-emitting regions associated with powerful radio galaxies, which often extend over several tens of kiloparsecs.

Since EELR are spatially resolved, we can deploy the powerful diagnostic tools of classical nebular analysis to map interactions between AGN and the interstellar medium (ISM) of their host galaxies and ultimately, perhaps, gain insight into the nature of the AGN phenomenon. First, however, we need to understand the mechanism by which EELR are ionized and excited. An AGN can influence its galactic environment either by means of high-energy radiation emitted by the primary continuum source, or by the collimated outflows of relativistic plasma which produce extended radio sources. Both of these are capable of causing ionization in the surrounding ISM and are invoked by the two main contending excitation models for EELR. I will briefly review these models and then outline the properties of various types of EELR, highlighting observational evidence which bears on the nature of the excitation mechanism.

\section{Ionization Mechanisms}

\subsection{The AGN Radiation Field}

Active galactic nuclei are strong ionizing continuum sources and any radiation that escapes the nucleus will photoionize the surrounding ISM. The Strömgren radius is $R_{s}=16.8 L_{46}^{1 / 3} n_{I S M}^{-2 / 3} \mathrm{kpc}$ where $L_{46}$ is the ionizing continuum luminosity in units of $10^{46} \mathrm{ergs} \mathrm{s}^{-1}$ and we assume a uniform interstellar medium (ISM) 
of average density $n_{I S M} \mathrm{~cm}^{-3}$. In reality, the ISM is far from uniform but this calculation nevertheless suggests that a typical quasar, emitting isotropically, is capable of of photoionizing the entire disk of a large spiral. Even a moderately luminous Seyfert should photoionize the ISM out to distances of several kiloparsecs. The spectra of EELR are indeed typically very similar to those of type 2 Seyferts and this, together with the success of simple photoionization models in reproducing the observed line ratios (e.g., Robinson et al. 1987), has encouraged the belief that most EELR are excited primarily by the AGN radiation field.

In simple photoionization models, the EELR is represented by a plane slab of gas of fixed density (or pressure), chemical composition, and thickness. For given values of these parameters the emission-line intensities are governed by the spectral energy distribution of the incident continuum and the ionization parameter, $U \propto F / n$, where $F=q / r^{2}$ is the ionizing photon flux, $n$ the density and $q$ the ionizing photon luminosity per steradian. The relative intensities of most of the strong optical emission lines are reasonably well reproduced by models in which the ionizing continuum is described either by a moderately steep power-law (e.g., $f_{\nu} \propto \nu^{-1.5}$ ) or EUV-emitting thermal sources, such as a hot blackbody or an accretion disk. The incoming continuum flux is subject not only to geometrical dilution but potentially also to attenuation by absorption or scattering in intervening material. Hence an inescapable prediction of AGN photoionization is that there should be strong radial gradients in either the ionization parameter or the gas density, or both.

The AGN radiation field is likely to be anisotropic on the size scales occupied by EELR. This may result from 'shadowing' by the dusty molecular torus which, according to 'unified models' (e.g., Antonucci 1993) encloses the primary continuum source, or the continuum may itself be emitted anisotropically. Intrinsic anisotropy could arise through limb-darkening if the EUV continuum is emitted by the photosphere of an accretion disk (Acosta-Pulido et al. 1990) or, in powerful radio galaxies, by Doppler beaming of UV synchrotron radiation emitted by the jets. The perceived morphology of EELR is therefore likely be determined by the intersection of the continuum beam with the surrounding gas distribution; the 'ionization cones' observed in several Seyferts (Wilson \& Tsvetanov 1994) are believed to be examples of anisotropic illumination of the ISM. Given that the continuum beam is co-axial with the radio jet we expect the EELR to be approximately aligned with the radio source.

If AGN photoionization is the dominant excitation mechanism, EELR can be used to estimate the ionizing luminosity of the central continuum in directions other than the direct line of sight, thereby allowing the degree of anisotropy to be inferred. A lower limit on the ionizing photon luminosity can be determined from the Balmer-line fluxes or, if $U$ and $n$ can be inferred from spectral diagnostics, $q$ can be estimated to within a geometrical projection factor (Penston et al. 1990). Conversely, since the continuum is likely to be anisotropic, an apparent deficit of ionizing photons does not necessarily rule out AGN photoionization.

Although simple photoionization models are broadly successful they also have some significant shortcomings. Notably the relatively high electron temperatures commonly inferred from the $[\mathrm{O}$ III $] \lambda 4363 /(\lambda \lambda 4959+5007)$ cannot be accounted for by these models (Tadhunter, Robinson, \& Morganti 1989; StorchiBergmann et al. 1996), nor can the models account for the observed strengths of high-ionization lines such as [Nev] $\lambda 3524$. This is, perhaps, not surprising given 
the simplistic plane slab geometry of such models. In reality, EELR spectra are likely to be emissivity-weighted averages of several (perhaps very many) clouds with a range of density and size. Better agreement with the $\left[\mathrm{O}_{\mathrm{III}}\right]$ temperature diagnostic and other line ratios can be achieved (with some fine tuning) by models incorporating a mixture of low-density matter-bounded and higher-density radiation-bounded clouds, with the former partially shielding the latter from the ionizing continuum (Binette et al. 1996).

\subsection{Fast Shocks}

Radio-emitting plasma is ejected from AGN in highly collimated relativistic jets as in powerful radio galaxies, or in sub-relativistic but supersonic plasma bubbles ('plasmons') as in Seyfert galaxies. In either case, violent dynamical interactions occur when this ejecta encounters the cool ISM. In particular, strong shocks driven into the cool gas cause local heating, ionization, and compression. For shock velocities $v_{s} \geq 200 \mathrm{~km} \mathrm{~s}^{-1}$, the gas is heated to temperatures in excess of $2 \times 10^{5} \mathrm{~K}$ and becomes a significant local source of ionizing radiation (Binette et al. 1985). Such photoionizing shocks have recently been discussed in detail as an ionization mechanism for EELR by Sutherland, Bicknell, \& Dopita (1993), Dopita \& Sutherland (1995) and Morse, Raymond, \& Wilson (1996). The ionizing radiation is absorbed both by the ambient gas upstream of the shock and the compressed gas downstream, once it has cooled and recombined sufficiently to build up a significant Lyman-continuum optical depth. For a plane shock propagating into a uniform medium, three distinct line-emitting zones can be identified: gas which is cooling and recombining after having been collisionally ionized by the shock; photoionized compressed gas further downstream; and a low-density photoionized precursor ahead of the shock.

The ionizing flux generated by the hot gas is a steep function of the shock velocity (roughly proportional to $v_{s}^{3}$ ) and hence for faster shocks, the photoionized regions dominate the line emission. The photoionized precursor will have a relatively high ionization parameter compared to that of the compressed downstream gas. The line intensity ratios are mainly governed by the shock velocity (which determines both the flux and the high-energy cut-off of the ionizing spectrum), the ambient density, and the magnetic field (which limits the post-shock compression and hence determines the ionization parameter in the downstream gas).

With shock velocities $v_{s} \approx 300-500 \mathrm{~km} \mathrm{~s}^{-1}$, emission-line intensities characteristic of Seyfert 2 nuclei are obtained (Dopita \& Sutherland 1995). This is essentially because at these velocities, the ionizing spectrum produced by the shock has a rather similar shape (an EUV bump with an exponential decline beyond a few hundred $\mathrm{eV}$ ) to that expected from an AGN accretion disk. In fact, the mean ionizing photon energy for $v_{s} \approx 300 \mathrm{~km} \mathrm{~s}^{-1}$, is $36 \mathrm{eV}$, very similar to continuum energy distributions used in successful AGN photoionization models.

As with AGN photoionization models, geometry is a major uncertainty. The predicted line intensities are likely to depend in detail on complexities such as clumpiness in the ambient medium and fragmentation in the downstream flow, or the variation in effective shock velocity around a bow-shock.

Radio jets are capable of efficiently transporting energy over distances of several tens of $\mathrm{kpc}$ from the AGN. On colliding with a sufficiently dense cloud, 
part of the jet's bulk kinetic energy will be thermalized and radiated locally as thermal continuum and line emission. In contrast to AGN photoionization, therefore, both the density and the excitation state of the line-emitting gas should peak near the site of the jet-cloud interaction. Signatures of such interactions include spatial associations between the line emission and radio knots or hot-spots, and evidence of a highly disturbed velocity field, such as steep gradients, line broadening or splitting.

This mechanism predicts a strong relationship between EELR ionization and shock velocity. Unfortunately it is not easy to determine the latter since the optical emission lines come from undisturbed gas upstream of the shock, or cooled gas downstream, and their kinematics will not necessarily reflect the shock speed. For the same reason, the spatial correspondence between the EELR and the radio source will not be precise.

\section{Properties of EELRs}

\subsection{The Resolved Narrow-Line Region in Seyfert Galaxies}

Recent HST observations of the narrow-line region (NLR) in Seyfert galaxies are reviewed by Wilson (this volume). However, it is worth noting here that the combination of $H S T$ images with high spatial-resolution radio maps is forcing a major revision of our understanding of the NLR, at least in those objects with extended radio sources. Striking spatial associations between line emission and radio structures have been revealed in several objects, providing compelling evidence for strong dynamical interactions between the emission line gas and radio-emitting ejecta on scales of $\leq 1 \mathrm{kpc}$. Maps of Mkn 573 (Capetti et al. 1996), for example, show arc-like emission-line structures bordering the doublelobed radio source. Such structures can be explained if the NLR is formed by the ambient ISM being swept up and compressed either as a result of the supersonic expansion of radio-emitting plasmons (Pedlar, Dyson, \& Unger 1985), or by bow-shocks around plasmons ejected supersonically from the nucleus (Taylor, Dyson, \& Axon 1992). In these models the swept-up gas is assumed to be photoionized by the AGN continuum but it is also possible, as noted in $\S 2.2$, that the plasmon-driven shocks themselves play an important role in ionizing the compressed gas (Dopita \& Sutherland 1995; also Metz et al. and Allen et al., this volume). Even if shocks are unimportant as ionization sources, it would seem that the bi-polar morphology of the NLR in these objects owes more to the influence of the radio source than to anisotropic illumination by the AGN continuum.

\subsection{The Extended (Very) Narrow-Line Region in Seyfert Galaxies}

In some Seyfert galaxies, high-ionization line emission extends over distances of several kiloparsecs from the nucleus, well beyond both the classical NLR and the nuclear radio source. These so-called extended narrow-line regions (ENLR) nevertheless tend to be elongated along the radio-source axis (Unger et al. 1987). They are kinematically distinct from the NLR, having much smaller line widths $\left(\lesssim 50 \mathrm{~km} \mathrm{~s}^{-1}\right.$ ) and velocities generally consistent with galactic rotation curves. These properties led Unger et al. to propose that the ENLRs are formed by 
photoionization of the normal galactic ISM by an anisotropic radiation field escaping from the AGN.

This hypothesis is broadly supported by detailed studies of individual objects. For example, there is little doubt that the spectacular sharp-edged bi-polar ENLR of NGC 5252 (Tadhunter \& Tsvetanov 1989) is indeed photoionized by a bi-conical radiation beam emerging from the nucleus (Prieto \& Friedling 1993; Wilson \& Tsvetanov 1994; Acosta-Pulido et al. 1996).

In NGC 4151, the radial density and ionization structure of the ENLR out to a distance of $2 \mathrm{kpc} \mathrm{SW}$ of the nucleus provide compelling evidence for AGN photoionization (Robinson et al. 1994). There is a sharp boundary between the NLR and ENLR marked by a decrease in line width, a factor of 10 drop in density and a corresponding increase in the inferred ionization parameter. Further out, the density remains approximately constant $\left(n_{e} \approx 200 \mathrm{~cm}^{-3}\right)$ while the ionization parameter declines at a rate consistent with geometrical dilution of the AGN radiation field. In this case, the morphology of the ENLR has the appearance of a knotty jet rather than a cone, and its axis is misaligned by nearly $30^{\circ}$ with respect to the radio source. However, these facts can easily be understood in terms of a conical radiation beam that is co-axial with the radio jet if the beam has a large opening angle $\left(\sim 120^{\circ}\right)$ and intercepts the galactic disk at grazing incidence (Vila-Vilaró 1993; Pedlar et al. 1993). The kinematics of the ENLR unambiguously indicate that it is part of the galactic disk. The velocity field of the ionized gas precisely matches the global rotation curve as derived from 21-cm measurements (Pedlar et al. 1992) and furthermore, high-resolution spectra show velocity splitting in $[\mathrm{O} \mathrm{II}] \lambda \lambda 4959,5007 \mathrm{SW}$ of the nucleus (Asif et al. 1996), with the two components straddling the range of 21$\mathrm{cm} \mathrm{H}$ I velocities measured at the same location. The $[\mathrm{O} I I I]$ components are symmetrically displaced from the velocity at the peak $\mathrm{H}$ intensity by an offset of $17 \mathrm{~km} \mathrm{~s}^{-1}$, consistent with the sound speed corresponding to the electron temperature in the ENLR $\left(T_{e} \approx 1.7 \times 10^{4} \mathrm{~K}\right.$; Robinson et al. 1994). A plausible interpretation of these results is that we are observing a photoionized outer skin expanding away from giant molecular clouds immersed in the AGN radiation field.

\subsection{Extended Emission-Line Regions in Radio Galaxies}

Extended emission-line regions are often found around powerful radio galaxies (Tadhunter 1986; Baum et al. 1988), and are also associated with some radioloud quasars (e.g., Stockton \& MacKenty 1987). These nebulae tend to be larger and more complex than the Seyfert ENLRs, with the ionized gas being distributed in knots, arc-like features or filaments over scales $\gtrsim 10 \mathrm{kpc}$. Despite the diverse and often complex morphologies, there is a statistical tendency for the line-emitting gas to align with the radio axis, although unlike Seyfert ENLRs, this gas rarely extends beyond the radio source (which often spans several hundred kiloparsecs). On the other hand, the emission-line spectra of radio-galaxy EELRs are also broadly characteristic of photoionization by an AGN-like continuum (Robinson et al. 1987).

In several sources there are clear morphological and kinematical signatures of dynamical interactions between radio jets and line-emitting gas (e.g., Heckman, van Breugel, \& Miley 1984; Clark 1996). It may well be that in these 
interactions the line-emitting gas is ionized in situ by photoionizing shocks, as advocated for Centaurus A by Sutherland et al. (1993), perhaps with contributions from other processes associated with the radio plasma such as relativistic particle heating (Ferland \& Mushotzky 1984), or locally produced synchrotron radiation (van Breugel et al. 1985).

Nevertheless, the AGN radiation field is presumably still present even in these cases and the balance between local and central-source ionization is unclear. It seems unlikely that all radio-galaxy EELR can be attributed solely to jet-cloud interactions: the emission-line gas is not always closely associated with the radio source, and its velocity field is often consistent with relatively unperturbed orbital motion (Tadhunter, Fosbury, \& Quinn 1989, Baum, Heckman, \& van Breugel 1992). In at least one case, the high-ionization emission-line knot associated with PKS 2152-69, there is convincing evidence for photoionization by beamed radiation from the nucleus (Tadhunter et al. 1988). This feature lies along the radio-source axis and is co-spatial with a highly polarized blue continuum source which cannot easily be explained either by shocks or local synchrotron production. It seems most likely that this is due to scattering of beamed continuum radiation from the nucleus by dust mixed with the emissionline gas (di Serego Alighieri et al. 1988). Thus, it is possible that jet-cloud interactions may sometimes simply compress ambient gas which is then 'lit up' by the AGN radiation field.

Perhaps the most spectacular known example of a dynamical interaction between radio jets and line-emitting gas is found in PKS 2250-41, a narrow-line radio galaxy at a redshift of $z=0.308$ with a powerful FR II radio source (Tadhunter et al. 1994). Line emission extends $40-60 \mathrm{kpc}$ either side of the nucleus, and is distributed in huge arc structures associated with radio lobes. The radio and optical structures are remarkably similar to those recently discovered in some Seyfert NLRs ( $\$ 3.1$ ), though on a much larger scale. In particular, the arc of line emission bordering the leading edge of the western radio lobe is strongly reminiscent of a bow-shock being driven into the ambient medium, as in the NLR model of Taylor et al. (1992).

A recent spectroscopic study (Clark et al. 1996) reveals that the ionization state as measured by the $[\mathrm{O} \mathrm{II}] \lambda 3727 /\left[\mathrm{O}_{\mathrm{III}}\right] \lambda 5007$ ratio has a pronounced minimum at the outer edge of the western radio lobe, while $[0 \mathrm{III}] \lambda 5007$ is also significantly narrower than low-ionization lines such as [N $\mathrm{NI}] \lambda 6584$ at this location. A possible explanation for these observations in terms of photoionizing shocks is that the high-ionization lines are produced mainly in the relatively undisturbed precursor, while low-ionization lines are emitted by the compressed, accelerated and probably turbulent gas downstream of the shock. The differences in line width could also result from illumination of the shock-induced gas flows by the AGN radiation field but this would not explain the arc-like morphology of the [O III] emission if it comes from gas which has not yet been swept up by the shock. Clark et al. argue that other characteristics of the emission line spectrum also favor shock models, notably the low He II $\lambda 4686 / \mathrm{H} \beta$ ratio and the relatively high [O III] temperature. However, as noted in $\$ 2.1$, these problems are alleviated in photoionization models which include both matter- and radiation-bounded clouds.

For the EELR associated with the $\mathrm{W}$ lobe to be photoionized by the AGN, a luminous quasar $\left(q>10^{55}\right.$ photons s $\left.^{-1}\right)$ would have to be hidden in the center of 
PKS 2250-41. Given the radio luminosity of this source, this can just about be accommodated within unified schemes which seek to identify FR II radio galaxies with radio-loud quasars (e.g., Barthel 1989).

In PKS 2250-41, therefore, we have compelling evidence for a violent dynamical interaction between the radio jets and the surrounding cool gas (Clark et al. propose that the cool gas is in fact the ISM of a companion galaxy). However, even in this case it is difficult to establish unambiguously whether the emission lines are excited locally by photoionizing shocks, or by the radiation field of a hidden quasar nucleus.

\subsection{The Alignment Effect in High-Redshift Radio Galaxies}

Extended line emission is also common around more distant $(z>0.5)$ radio galaxies and is associated with elongated, often multi-component, UV-continuum structures which are tightly aligned with the radio source axis (McCarthy et al. 1987; McCarthy 1993). The cause of this 'alignment effect' is a contentious issue. One hypothesis is that shocks induced by the radio jet compress the ambient gas and trigger star formation (e.g., Rees 1989). Alternatively, Doppler-beamed radiation from a hidden quasar nucleus could photoionize gas along the radio axis, with the extended blue continuum being light scattered out of the beam by dust or electrons (Tadhunter, Fosbury, \& di Serego Alighieri 1989; Fabian 1989). The latter explanation is strongly supported by polarization measurements of several objects which reveal the UV continuum to be polarized at levels greater than $10 \%$, with $E$-vectors roughly perpendicular to the radio-source axis, as would be expected if the light is scattered (e.g., Cimatti et al. 1993).

Nevertheless, significant polarization has so far been detected in only a minority of the high- $z$ radio galaxies, and it remains unclear whether these objects are typical or whether other mechanisms dominate in other sources. Apart from jet-induced starbursts, the line emission and extended UV continuum might arise from fast photoionizing shocks and, regardless of the dominant ionization mechanism, the nebular continuum of the line-emitting gas can itself contribute a substantial fraction of the extended continuum emission (Dickson et al. 1995).

Acknowledgments. I am grateful to the IAU and the Royal Society for financial support and also to Neil Clark and Clive Tadhunter for communicating their results in advance of publication.

\section{References}

Acosta-Pulido, J. A., et al. 1990, ApJ, 365, 119.

Acosta-Pulido, J.A., et al. 1996, ApJ, 464, 177.

Antonucci, R. R. J. 1993, ARA\&A, 31, 473.

Asif, M., et al. 1996, MNRAS, submitted.

Barthel, P. D. 1989, ApJ, 336, 606.

Baum, S. A., et al. 1988, ApJS, 68, 643.

Baum, S. A., Heckman, T. M., \& van Breugel, W. J. M. 1992, ApJ, 389, 208.

Binette, L., Dopita, M. A., \& Tuohy, I. R. 1985, ApJ, 297, 476.

Binette, L., Wilson, A.S., \& Storchi-Bergmann, T. 1996, A\&A, in press. 
Capetti, A., et al. 1996, ApJ, in press.

Cimatti, A., et al. 1993, MNRAS, 264, 421.

Clark, N.E. 1996, Ph.D. Thesis, University of Sheffield.

Clark, N.E., et al. 1996, MNRAS, submitted.

Dickson, R. 1995, MNRAS, 273, 29P.

di Serego Alighieri, S., et al. 1988, Nat., 334, 591.

Dopita, M.A., \& Sutherland, R. S. 1995, ApJ, 455, 468.

Fabian, A.C. 1989, MNRAS, 238, 41P.

Ferland, G.J., \& Mushotzky, R. F. 1984, ApJ, 286, 42.

Heckman, T.M., van Breugel, W. J. M., \& Miley, G. K. 1984, ApJ, 286, 509.

McCarthy, P. J., et al. 1987, ApJ, 321, L29.

McCarthy, P. J. 1993, ARA\&A, 31, 639.

Morse, J. A., Raymond, J. C., \& Wilson, A.S. 1996, PASP, $108,426$.

Pedlar, A., Dyson, J. E., \& Unger, S. W. 1985, MNRAS, 214, 463.

Pedlar, A., et al. 1992, MNRAS, 259, 369.

Pedlar, A., et al. 1993, MNRAS, 263, 471.

Penston, M. V., et al. 1990, A\&A, 236, 53.

Prieto, M. A., \& Freudling, W. 1993, ApJ, 418, 668.

Rees, M.J. 1989, MNRAS, 239, 1P.

Robinson, A., et al. 1987, MNRAS, 227, 97.

Robinson, A., et al. 1994, A\&A, 291, 351.

Stockton, A., \& MacKenty, J.W. 1987, ApJ, 316, 584.

Storchi-Bergmann, T., et al. 1996, A\&A, in press.

Sutherland, R.S., Bicknell, G. V., \& Dopita, M. A. 1993, ApJ, 414, 510.

Tadhunter, C. N. 1986, D. Phil. thesis, University of Sussex.

Tadhunter, C. N., et al. 1988, MNRAS, 235, 403.

Tadhunter, C. N., \& Tsvetanov, Z. 1989, Nat., 341, 422.

Tadhunter, C. N., Fosbury, R. A.E., \& Quinn, P. J. 1989, MNRAS, 240, 225.

Tadhunter, C.N., Fosbury, R. A.E., \& di Serego Alighieri, S. 1989, in BL Lac Objects, ed. L. Maraschi, T. Maccacaro \& M.-H. Ulrich (Springer-Verlag: Berlin), 79.

Tadhunter, C.N., Robinson, A., \& Morganti, R. 1989, in Extranuclear Activity in Galaxies, ed. R. A. E. Fosbury, \& E. J.A. Meurs (ESO Conference and Workshop Proceedings No. 32), 293.

Tadhunter, C.N., et al. 1994, A\&A, 288, L21.

Taylor, D., Dyson, J. E., \& Axon, D. J. 1992, MNRAS, 255, 351.

Unger, S. W., et al. 1987, MNRAS, 228, 671.

van Breugel, W., et al. 1985, ApJ, 290, 496.

Vila-Vilaró, B. 1993, Ph.D. Thesis, University of La Laguna.

Wilson, A.S., \& Tsvetanov, Z. I. 1994, AJ, 107, 1227. 